\title{
Die Deregulierung der US-Finanzmärkte als eine Ursache ihrer Krise
}

\begin{abstract}
Stefan Schiman*
Zusammenfassung

Die Finanzmarktreformen nach der Depression in den 1930er-Jahren und die Neuordnung des internationalen Finanzsystems in Bretton Woods 1944 bildeten die Grundlage für eine marktwirtschaftliche Ordnung mit stark regulierten Finanzmärkten, die sich im Westen nach dem Zweiten Weltkrieg etablierte. Die Instabilität des Wechselkursregimes aufgrund der asymmetrischen Rolle des Dollars bewirkte den Zusammenbruch dieses Ordnungsrahmens. Starken Wechselkursschwankungen folgten Erdölpreisschocks, die eine Hochzinspolitik auslösten, welche die Regulierung der Finanzmärkte in den USA untergrub. Die durch die Erdölkrise beschädigte nachfrageorientierte Wirtschaftspolitik wurde zudem von einer neoliberalen Doktrin abgelöst, die den Deregulierungsprozess theoretisch fundierte. Der mit dessen kompetitivem Charakter einhergehende Druck auf geschützte Finanzdienstleistungen und auf deren Profitabilität erzeugte eine Welle an Zusammenschlüssen in der Finanzwirtschaft und ermöglichte den dadurch entstandenen Finanzkonglomeraten immer riskantere Geschäfte, die mit dem Einlagen- und Kreditgeschäft eng verzahnt waren. Diese Gemengelage, die an die Ursprünge der Finanzmarktkrise 1929 erinnert, entzündete sich schließlich am Immobilienpreisboom in den USA und mündete in die Finanzmarktkrise 2007/o8.
\end{abstract}

Schlagwörter: Finanzmarktkrise, Finanzmarktderegulierung, Zinssatzdeckelung, Trennbankensystem, Financial Repression

\section{The Deregulation of US Financial Markets as a Cause of Their Crisis} Abstract

Financial market reforms in the wake of the Great Depression of the 1930 and the reshaping of the international financial system in Bretton Woods 1944 laid the foundations of a market-based economic framework with highly regulated financial markets that was established in the Western hemisphere after the Second World War. The instability of the exchange rate regime due to the asymmetric role of the dollar led to the breakdown of this regulatory framework. Strong exchange rate volatility was followed by oil price shocks which gave rise to a policy of high interest rates that undermined financial market regulation in the USA. The demand-oriented macroeconomic policy, no longer sustainable after the oil crisis, was superseded by a neo-liberal doctrine that provided the theoretical underpinning to the deregulation process. Its implicit competitive stance put pressure on sheltered financial services and their profitability, leading to a wave of mergers in the financial sector; these large financial groups were able to embark on ever more risky operations that were closely entangled with the deposit and loan business. This mixture, akin to the origins of the financial market crisis of 1929, ignited with the real estate price boom in the USA and triggered the financial market crisis of 2007-08.

Keywords: financial market crisis, financial market deregulation, interest rate cap, Glass-Steagall reform, financial repression

\footnotetext{
*Stefan Schiman, WIFO. E-Mail: Stefan.Schiman@wifo.ac.at.

Der Autor dankt Paul Ramskogler, Stephan Schulmeister, Gunther Tichy und zwei anonymen Gutachtern für wertvolle Kommentare und Martha Steiner für die wissenschaftiche Assistenz.
} 


\section{Einleitung}

Die volkswirtschaftlichen Entwicklungen, die mit der Finanzmarktkrise 2007/08 in Verbindung gebracht werden, sind vielfältig: Sie erstrecken sich von den Ungleichgewichten im Außenhandel über verzerrte Anreize im Wertpapierrating bis zu den Auswüchsen des Schattenbankwesens und den Übertreibungen am amerikanischen Häusermarkt (vgl. Ramskogler 2015; Schuberth 2013; Beirat für Wirtschafts- und Sozialfragen 2009; Url 2009). Diese Vielfalt illustriert die weitreichenden Verzweigungen der Krise und ihres Zustandekommens. Im historischen Blickfeld stellt sich die Finanzmarktkrise als Kulminationspunkt eines Prozesses dar, der in den 1970er-Jahren seinen Ausgang nahm und die Weltwirtschaft seither entscheidend geprägt hat: der Deregulierung der Finanzmärkte (Tichy 2014: 224-225; Bhidé 2009; Stiglitz 2009¹). Einige aktuelle Beiträge zur politisch-ökonomischen Literatur arbeiten den Zusammenhang zwischen der Finanzmarktkrise und der Deregulierung der Finanzmärkte (vor allem in den USA) besonders deutlich heraus (Kotz 2013; Silvers 2013; Stockhammer 2013; Wolfson 2013).

Wie die Finanzmarktkrise nur im Kontext der Deregulierung zu verstehen ist, kann der Prozess der Deregulierung selbst nur vor dem Hintergrund der Finanzmarktregulierung und des sie umgebenden Ordnungsrahmens verstanden werden. Für die weitreichende Regulierung, insbesondere in dem für die Entwicklung der Finanzmärkte maßgeblichen angloamerikanischen Raum, waren zwei zentrale historische Ereignisse des 20. Jahrhunderts bestimmend (Wolfson 2013: 180). Das erste, die Große Depression 1929-1933, war ihrerseits die Folge von Übertreibungen an den Finanzmärkten (Eichengreen 2015: 3). Aufgrund der augenscheinlichen Parallelen zur Großen Rezession 2008/09 ${ }^{2}$ wird sie hier als historischer Ausgangspunkt gewählt, dem unmittelbar eine Epoche überwiegend

1 Für eine quantitative Analyse des Zusammenhangs zwischen Finanzmarktderegulierung und Finanzmarktkrise vgl. Bentley 2015.

2 Was die unmittelbare wirtschaftspolitische Reaktion betrifft, unterscheiden sich die beiden Wirtschaftskrisen maßgeblich voneinander: Während die Große Depression durch restriktive Budget- und Einkommenspolitiken und protektionistische Maßnahmen verstärkt wurde, war die Wirtschaftspolitik 2008/o9 viel stärker von den Einsichten geprägt, die Keynes durch die wirtschaftspolitischen Irrungen seinerzeit gewonnen hatte. regulierter Finanzmärkte folgte (bis in die 1970erJahre). Neben der Großen Depression war das zweite für die Beschaffenheit des Währungssystems und der Kapitalmärkte bestimmende Ereignis der Zweite Weltkrieg 1939-1945. Er erzeugte große Verwerfungen in der Industrielandschaft der Achsenmächte, die einen Wiederaufbau erforderlich machten, der stark von öffentlichen Interessen geprägt war und Regulierungen ein günstiges Umfeld bot (Eichengreen 2007: 91-92, 95). Außerdem führte der Krieg zu einem starken Anstieg der Staatsverschuldung, deren Rückführung unter anderem durch Maßnahmen der Finanzmarktregulierung erzielt wurde („Financial Repression“, Reinhart/ Sbrancia 2015: 299). Darüber hinaus erzeugte er ein günstiges Umfeld für Kooperation und Zusammenarbeit, auf dem sich das auf Koordination und Finanzmarktregulierung beruhende Weltwährungssystem von Bretton Woods etablieren konnte (Helleiner 2010: 621). Dieses war aber nicht nur ein Resultat kooperativen Verhaltens der Nationen, sondern vor allem ein Abbild des hegemonialen Anspruchs der USA, der seinen Ausdruck in der Sonderrolle des US-Dollars als Ankerwährung fand. Die Ausgestaltung des Währungssystems führte letztlich zu dessen Scheitern (Schulmeister 2000: 365) und in weiterer Folge zum sukzessiven Abbau der Finanzmarktregulierung (Silvers 2013: 433).

Im folgenden, zweiten Abschnitt werden die zentralen Regulierungsmaßnahmen im Rahmen der NewDeal-Finanzmarktreformen und die Funktionsweise von „Financial Repression“ behandelt. Der dritte Abschnitt erläutert die Einbettung der Finanzmarktregulierung in den marktwirtschaftlichen Ordnungsrahmen von Bretton Woods. Wie nach dessen Zusammenbruch die Finanzmarktderegulierung in Gang kam, zeigt der vierte Abschnitt. Der fünfte beschreibt den Übergang vom keynesianischen zum neoliberalen Paradigma in den 1970er-Jahren, der in den Prozess der kompetitiven Deregulierung ab den 1980er-Jahren mündete. In Abschnitt sechs wird gezeigt, dass sich durch die zunehmende Entfesselung der Finanzmärkte riesige Finanzkonglomerate bildeten, deren Transaktionen und Finanzinnovationen aufs Engste miteinander verwoben waren und die sich schließlich im Epizentrum der Finanzmarktkrise 2007/o8 wiederfanden. ${ }^{3}$

3 Die Geschichte der Finanz- und Kapitalmarktregulierung wurde oft durch bewusste politische Entscheidungen geprägt (vgl. Helleiner 1994, Levine 2012). In diesem Artikel soll gezeigt werden, wodurch die Deregulierungspolitik der letzten Jahrzehnte begünstigt wurde. 
2. New Deal und „Financial Repression“ als Grundlagen der Finanzmarktregulierung

\subsection{Die Finanzmarktreformen des New Deal}

Der Banking Act von 1933 (auch: Glass-Steagall-Act) war eine wirtschaftspolitische Reaktion auf die Große Depression in den USA und deren Ursachen. Die damit einhergehende umfassende Regulierung des amerikanischen Finanzwesens wurde zu einem integralen Bestandteil eines neuen Ordnungsrahmens, der die Volkswirtschaft der USA und die Weltwirtschaft in den folgenden Jahrzehnten prägte (Eichengreen 2015: 244ff. und 318). Im Wesentlichen sind mit dem Banking Act folgende Maßnahmen verbunden (Wolfson 2013: 179-180):

- die Schaffung einer Einlagensicherung,

- die institutionelle Trennung von Einlagen- und Wertpapiergeschäft („Spartentrennung“),

- die Deckelung der Zinssätze.

Die Einlagensicherung entkoppelte die Sicherheit der Bankeinlagen (bis zu einer gewissen Höhe) vom wirtschaftlichen Schicksal der jeweiligen Bank. Dadurch wurde der Teufelskreis aus gegenseitigem Misstrauen an den Finanzmärkten unterbrochen und Bank-Runs wurden unterbunden. Die Spartentrennung sah wiederum eine Trennung von Geschäfts- und Investmentbanken vor, die den Kreditkreislauf der Realwirtschaft von den Vermögensmärkten entkoppelte. Regulation $Q$ bewirkte eine Deckelung der Zinssätze auf Spareinlagen und ein Zinsverbot für Sichteinlagen. Ein Ziel war es, den Preiswettbewerb unter den Anbietern, also den Wettbewerb der Banken um Kundeneinlagen über höhere Zinssätze, zu verringern (Gilbert 1986: 23). Die Elemente der New-Deal-Finanzmarktreform ergänzten einander; so wirkte die Zinssatzregulierung der Profitdämpfung, die die Spartentrennung verursachte, entgegen. Es ist daher nicht verwunderlich, dass auf die Beendigung der Zinssatzdeckelung in der Frühphase der Deregulierung in den 1980er-Jahren die sukzessive Aushöhlung der Spartentrennung folgte.

Ein weiterer Aspekt von Finanzmarktregulierung ist, dass sie den Zuwachs der Vermögenseinkommen schmälert. Der Erfolg der Deregulierung ab den 1980er-Jahren beruhte nicht zuletzt darauf, dass auch breitere Bevölkerungsschichten durch kapitalgedeckte Vorsorgesysteme von einem besseren Zugang zu ertragreicheren (und riskanteren) Anlageformen - vermeintlich - profitierten (Gilbert 1986: 29-30; Enkyo 1989: 101), während das gesamtwirtschaftliche Risiko deregulierter Finanzmärkte mit dem wachsenden zeitlichen Abstand zur Großen Depression immer stärker in den Hintergrund trat (Silvers 2013: 433).

\subsubsection{Zinssatzdeckelung - Stabilisierung des Finanzsystems}

Die Zinssatzdeckelung wurde in den USA in Form der Regulation Q im Rahmen des Banking Act 1933 eingeführt. Sie sah ein Verbot von Zinssätzen auf Sichteinlagen vor sowie eine Deckelung jener auf Spareinlagen bei Geschäftsbanken. Für gemeinnützige Bausparkassen („savings and loan associations“) wurde die Deckelung ab 1966 etwas höher angesetzt, um den privaten Wohnbau zu fördern (Eichengreen 2015: 68).

Mit der Deckelung der Einlagenzinssätze wurden mehrere wirtschaftspolitische Ziele verfolgt (Gilbert 1986: 22ff.): Erstens sollte damit ein aggressiver Wettbewerb um Kundeneinlagen unterbunden werden. Im Vorfeld der Großen Depression übertrafen sich die Geschäftsbanken mit immer höheren Einlagenzinssätzen, da das Kreditangebot in der Endphase des Kreditbooms kaum mit der steigenden Nachfrage Schritt hielt. Die Deckelung war ein Versuch, eine neuerliche Kreditblase zu unterbinden, indem deren Finanzierung erschwert wurde. Ein weiteres Ziel von Regulation Q war, die Rentabilität von Finanzanlagen zu senken und so Kredite stärker in realwirtschaftliche Investitionen zu lenken. Banken sollten angehalten werden, weniger in Finanzprodukte zu investieren und mehr Kredite an den Nichtbankensektor (an die Realwirtschaft) zu vergeben. Dies sollte zudem die Forderungen der Banken untereinander reduzieren und so - als drittes Ziel - die Anfälligkeit des Interbankenmarkts für Vertrauenseffekte verringern, sodass er in Krisensituationen weniger rasch zum Erliegen kommt. Hinsichtlich der Stabilisierung des Finanzsystems war die Regulierung im Großen und Ganzen erfolgreich, wie die Finanzmarktkrise von 2007/o8 kontrafaktisch zeigt: Der Deregulierung seit den $1980 e r-J a h r e n$ folgte genau jene enge Verflechtung innerhalb der Finanzwirtschaft, die für die Tiefe der späteren Krise letztlich eine wichtige Rolle spielte.

Neben den Zielen, das Entstehen von Kreditblasen zu verhindern, die Attraktivität von Finanzinvestitionen zu verringern und das Finanzwesen langfristig zu stabilisieren, wurde mit der Zinssatzdeckelung aber auch die Absicht verfolgt, die Geschäftsbanken für Erschwernisse, die sie im Zuge des Banking Act tragen mussten, zu kompensieren: Aufgrund der Spartentren- 
nung nahm die Profitabilität der Banken ab, da Netzwerkeffekte wegfielen. Die Zinssatzdeckelung erhöhte dagegen tendenziell die Profitabilität der Banken auf Kosten der Vermögenseinkommen der Sparer.

\section{2 „Financial Repression“ im Lichte der Finanz- marktregulierung}

Aus Sicht der privaten Haushalte handelte es sich bei der Zinssatzdeckelung also um eine Beschränkung ihrer Vermögenseinkommenszuwächse. Dieser Effekt wurde durch spätere Regulierungsmaßnahmen, die in der Literatur als „Financial Repression“ bezeichnet werden (vgl. Reinhart/Sbrancia 2015; Battilossi 2005), verstärkt. Es handelt sich dabei im Wesentlichen um die Besserstellung heimischer Anlagemöglichkeiten (insbesondere Staatsanleihen) gegenüber Veranlagungen im Ausland durch:

- Kapitalverkehrskontrollen,

- hohe Reservevorschriften für Banken,

- variierende Hinterlegungsvorschriften je nach Veranlagungsdestination,

- Finanztransaktionssteuern.

Derlei Maßnahmen prägten den finanzpolitischen Ordnungsrahmen der Industrieländer über mehrere Jahrzehnte hinweg bis zum Einsetzen der Deregulierung in den 1980er-Jahren. Ähnlich wie Regulation Q dämpften sie die Vermögenszuwächse der Sparer. Während hingegen die Geschäftsbanken von Regulation Q profitierten, da ihre Finanzierungskosten gedeckelt wurden, war „Financial Repression“ eine Umverteilung des gesamtwirtschaftlichen Vermögenszuwachses zugunsten der öffentlichen Hand. Auf diese Weise wurde einer Entwicklung begegnet, die ursächlich mit dem Zweiten Weltkrieg (1939-1945) in Zusammenhang steht: der stark angestiegenen Staatsverschuldung (Reinhart/Sbrancia 2015: 294-295). In den USA bewegte sich die Staatsschuldenquote im Zeitraum zwischen dem Ende der Großen Depression 1933 und dem Eintritt in den Zweiten Weltkrieg 1941 stabil um $40 \%$ bis $45 \%$ des BIP. Innerhalb der folgenden fünf Jahre verdreifachte sie sich kriegsbedingt auf über 120 $\%$ des BIP.

Die wirtschaftspolitischen Maßnahmen von „Financial Repression“ führten dazu, dass die Veranlagung von Vermögen in öffentlichen Schuldverschreibungen gegenüber anderen Veranlagungsformen begünstigt wurde. Gleichzeitig erschwerte „Financial Repression“ den Sparern und den Banken den Zugang zu ertragreicheren Anlageformen und senkte so - ähn- lich wie Regulation Q - die durchschnittliche Rendite von Finanzanlagen (Reinhart/Sbrancia 2015: 301).

\section{Finanzmarktregulierung als Element der Bretton- Woods-Ordnung}

Bestimmend für die Regulierung der Finanzmärkte bis Anfang der 1980er-Jahre waren demnach zwei zentrale historische Ereignisse des 20. Jahrhunderts: die Große Depression 1929-1933 (damit verbunden: die Popularität des keynesianischen Gedankenguts) und der Zweite Weltkrieg 1939-1945. Die Finanzmarktregulierungen des New Deal waren eine unmittelbare Folge der Großen Depression; der Krieg wiederum erzeugte ein für Regulierung und wirtschaftspolitische Kooperation günstiges Umfeld. Daraus erwuchs ein wirtschaftspolitischer Ordnungsrahmen, der als „embedded liberalism“ (vgl. Ruggie 1982), „restrictive financial order“ (Helleiner 1994: 3ff.), „regulated capitalism“ (Kotz 2013: 396ff.) oder „Fordist accumulation regime“ (Stockhammer 2013: 513) bezeichnet wurde. Bestimmte Elemente der Wirtschaftsordnung wurden eingeschränkt (Freiheit des Kapitalverkehrs), um andere zu bewahren (Freihandel) und gleichzeitig neue wirtschaftspolitische Ansprüche (nationalstaatliche Spielräume in der Wirtschaftspolitik) einzulösen (Ruggie 1982: 385, 392). Es geht also um die Abwendung von einem Laissezfaire-Liberalismus hin zu einer marktwirtschaftlichen Ordnung, in der finanzwirtschaftliche Möglichkeiten zugunsten von realwirtschaftlichen Zielen beschränkt wurden (Ghosh/Qureshi 2016: 4, 15). Der Beginn dieser Epoche kann mit der Konferenz von Bretton Woods (1944) datiert werden, bei der die wesentlichen Eckpfeiler der künftigen internationalen Wirtschaftsordnung festgelegt wurden; einer Wirtschaftsordnung, die stark auf den keynesianischen Ideen der 1930er-Jahre beruhte und in der die Regulierung der Finanzmärkte eine zentrale Rolle einnahm.

Die wirtschaftspolitische Autonomie der Nationalstaaten, die in diesem neuen Ordnungsrahmen eine wichtige Stellung hatte, umfasste nicht nur fiskal- und geldpolitische Eingriffe im Sinne der Konjunkturpolitik. Für Regionen mit wirtschaftlichem Aufholbedarf, zu denen nach dem Zweiten Weltkrieg auch Westeuropa und Japan zählten, schloss es außerdem den Aufbau und die Förderung nationaler Industrien mit ein. Diese wirtschaftspolitischen Ansprüche wurden durch eine Beschränkung der freien Marktkräfte der Finanzmärkte eingelöst, die im Wesentlichen durch folgende Maßnahmen erzielt wurde: 
- Beschränkung des Kapitalverkehrs,

- Fixierung der Wechselkurse,

- Regulierung des Finanzsystems.

Dadurch wurden Zinssätze und Wechselkurse, zwei für die langfristige ökonomische Planbarkeit zentrale Vermögenspreise, reguliert. Effektive Kapitalverkehrsbeschränkungen unterbanden einen Deregulierungswettbewerb zwischen den Ländern, indem sie Ausweichverhalten erschwerten (Battilossi 2005: 11). Diese Komplementarität der ordnungspolitischen Eingriffe verlieh der Bretton-Woods-Ordnung eine gewisse Stabilität und die Finanzmarktregulierung erlangte damit eine tragende, systemisch relevante Rolle im wirtschaftspolitischen Ordnungsrahmen nach 1944.

Im Bretton-Woods-Wechselkursregime wurde der US-Dollar als Ankerwährung festgelegt. Sein Preis wurde in Goldeinheiten fixiert (35 \$ pro Feinunze) und die amerikanische Zentralbank verpflichtete sich zum unbeschränkten Umtausch von Dollar und Gold zum festgelegten Kurs. Im Gegensatz zum Goldstandard früherer Jahre galt diese Verpflichtung nur gegenüber anderen Zentralbanken; eine Einschränkung, die sich aus der Logik der Kapitalverkehrsbeschränkung für den privaten Sektor ergab. Mit dieser asymmetrischen Ausgestaltung des Wechselkursregimes, die letztlich zum Scheitern des Bretton-Woods-Systems führte und den Weg für die Deregulierung der Finanzmärkte bereitete, setzte sich die USA durch. Großbritannien hatte ein multilaterales Regime mit einer internationalen Währung (Bancor) und einer zentralen Clearingsstelle bevorzugt (Handler 2008: 5-6). Die USA trachteten hingegen danach, ihren hegemonialen Anspruch durch die Sonderstellung ihrer Währung zu festigen (Helleiner 1994: 14).

Im Gegensatz zur Ausgestaltung des Wechselkurssystems herrschte hinsichtlich der Rolle des Finanzsystems zwischen den beiden Hauptakteuren von Bretton Woods (die USA, vertreten durch Harry Dexter White, und Großbritannien, vertreten durch John Maynard Keynes) sowie den anderen Teilnehmerländern im Großen und Ganzen Übereinstimmung: Finanzdienstleistungen wurden nicht auf einer Ebene mit den realwirtschaftlichen Sektoren gesehen, sondern ihnen nachgeordnet (Helleiner 1994: 37). Das vorrangige wirtschaftspolitische Ziel war es, den materiellen Wohlstand für breite Bevölkerungsschichten zu erhöhen. Eingedenk der negativen Erfahrungen im Zuge der Großen Depression wurden unregulierte Finanzmärkte als schädlich erachtet. Eine wohlstandsmehrende marktwirtschaftliche Ordnung und ein unreguliertes Finanzsystem schlossen sich entsprechend der vorherrschenden keynesianischen Sichtweise aus. Diese Sichtweise prägte die folgenden zwei Jahrzehnte; Finanzdienstleistungen führten ein Schattendasein im wirtschaftlichen Fortschritt der Nationen.

\section{Aufkeimende Deregulierung in einem instabilen Währungssystem}

Im Schatten des beispiellosen Wachstums des materiellen Wohlstands in den aufholenden Industrienationen, aber auch in den USA, wird bereits in den 1950er-Jahren die Saat für die spätere Deregulierung der Finanzmärkte gelegt. In makroökonomischer Hinsicht wurzelte sie in der Asymmetrie des Wechselkursregimes bzw. der Doppelrolle des US-Dollars als nationale und als Reservewährung (Eichengreen 1996: 107). ${ }^{4}$ Die damit einhergehende starke Dollarnachfrage setzte bereits in der zweiten Hälfte der 1940er-Jahre ein, als die Unsicherheit über das wirtschaftliche Los Europas zu einer Kapitalflucht in die USA führte (Kapitalkontrollen waren $\mathrm{zu}$ dieser Zeit noch mangelhaft), die zum Teil durch die Marshall-Hilfe kompensiert wurde (Helleiner 1994: 58-59). Ein systematischerer Dollarengpass ergab sich durch das hohe weltweite Wirtschaftswachstum und die starke Zunahme des Außenhandels: Da die Zentralbanken veranlasst waren, ausreichend Dollarreserven zu halten, um die Stabilität der Wechselkurse ihrer Währungen gegenüber dem US-Dollar zu gewährleisten, beschleunigte sich die internationale Dollarnachfrage. In der amerikanischen Zahlungsbilanz führte dies zu dauerhaften Ungleichgewichten. Da die Ausweitung des Dollarangebots wegen der Goldpreisbindung limitiert war, wurden die hohen Kapitalimporte anderweitig ausgeglichen; z. B. durch den Kauf ausländischer Unternehmen oder durch Auslandshilfe, insbesondere den Marshallplan (Der Spiegel 1961: 19).

Mit dem Anstieg der weltweiten Dollarreserven sank jedoch gleichzeitig das Vertrauen in die USZentralbank, diese in unbeschränktem Maße gegen den fixen Betrag von 35 \$ je Feinunze zu tauschen, da die Goldvorräte beschränkt waren. Diese dynamische Instabilität des Bretton-Woods-Systems wird als „Triffin-Dilemma“ bezeichnet, benannt nach seinem Autor Robert Triffin: Es bezeichnet das Dilemma der amerikanischen Geldpolitik, einerseits die Weltwirtschaft mit ausreichend Dollarreserven $\mathrm{zu}$ versorgen, aber

4 Gleichzeitig entstanden wissenschaftliche Arbeiten, die die spätere Deregulierung theoretisch untermauerten (z.B. Friedman 1953). 
damit gleichzeitig den Goldwert des Dollars und das Vertrauen in die eigene Währung zu schwächen, oder andererseits die Geldmengenausweitung zu drosseln und eine Weltwirtschaftskrise auszulösen (vgl. Bordo 2017). Die Verbindlichkeiten der amerikanischen Zentralbank übertrafen 1960 erstmals die US-Goldreserven; es setzte zunehmend ein Umtausch von Dollarreserven in Gold ein und die Dollarknappheit kehrte sich in ein Überangebot um (Eichengreen 1996: 116). Die Dollarverkäufe hätten durch höhere Zinssätze gedrosselt werden können; eine restriktivere Geldpolitik hätte jedoch konjunkturdämpfende Effekte mit sich gebracht und daher nicht der noch vorherrschenden keynesianisch inspirierten Wachstumspolitik entsprochen. Der realwirtschaftliche Erfolg der Bretton-Woods-Ordnung (gemessen am hohen Wirtschaftswachstum) war damit gleichsam Vorbote seines Untergangs (vgl. dazu Kotz 2013: 398, 408).

\subsection{Der Eurodollarmarkt und die Konsolidie- rung der Finanzwirtschaft}

Im Lichte der zunehmenden weltweiten Dollarskepsis nahmen die USA Ende der 1950er-Jahre eine Entwicklung in Kauf, die in London ihren Ausgang hatte (Enkyo 1989: 57ff.): die Etablierung des Eurodollarmarkts. Am Eurodollarmarkt wurden außerhalb der USA und unter anderem von nichtamerikanischen Banken Kredite vergeben, die in US-Dollar denominiert waren. Der Eurodollarmarkt wurde nicht nur von kommunistischen Staaten genutzt, um Dollartransaktionen außerhalb der USA zu tätigen. Einen Popularitätsschub erhielt er auch durch die Pfundkrise 1957 bzw. die Kapitalverkehrskontrollen, die zu deren Eindämmung eingesetzt wurden (Helleiner 1994: 83-84); diese konnten nämlich durch das Ausweichen auf in US-Dollar denominierte Kredite umgangen werden.

Das wesentliche Merkmal des Eurodollarmarkts in Bezug auf das Verständnis von Finanzmarktderegulierung ist, dass es sich um einen höchst unregulierten Markt handelte. Eurodollarkredite unterlagen nicht den Mindestreserveanforderungen der amerikanischen Zentralbank, sie waren nicht durch die Einlagensicherung gedeckt und die Zinssatzdeckelung kam nicht zur Anwendung. Durch diesen Mangel an Regulierung wurden höhere Zinserträge erzielt. Dies wiederum stärkte die Dollarnachfrage ohne die konjunkturschädlichen Nebenwirkungen einer restriktiven Geldpolitik; darüber hinaus beeinträchtigte der unregulierte Eurodollarmarkt die Finanzmarktregulierung in den USA selbst (noch) nicht. Großbritannien sah in dessen Etablierung zudem die Möglichkeit, London als internationales Finanzzentrum zu bewahren.

Somit war der Anspruch auf eine keynesianischexpansive Wirtschaftspolitik innerhalb des asymmetrischen Ordnungsrahmens von Bretton Woods, verbunden mit den hegemonialen Ansprüchen der USA und den Ansprüchen Londons als internationales Finanzzentrum, mitbestimmend dafür, dass der Deregulierungsprozess „offshore“, also außerhalb nationalstaatlicher Regeln, in Gang gesetzt wurde. Die Handlungsmöglichkeiten des internationalen Finanzkapitals nahmen dadurch erheblich zu. Die Finanzwirtschaft konnte ihre gesamtwirtschaftliche Stellung, die sie vor der Weltwirtschaftskrise 1929 innegehabt und durch die Regulierung eingebüßt hatte, zunehmend konsolidieren.

Es zeigte sich aber, dass der Offshore-Markt für US-Dollar es auf die Dauer nicht vermochte, die fundamentalen Konstruktionsfehler von Bretton Woods, insbesondere das Triffin-Dilemma, auszugleichen. Die expansive Wirtschaftspolitik in den USA in den 196oerJahren, insbesondere steigende Militärausgaben für den Vietnamkrieg, aber auch steigende Sozialausgaben im Rahmen der „Great Society“-Initiative, verstärkten die Krisenanfälligkeit des Dollars (Helleiner 1994: 86). Der Rückgang der US-Goldreserven setzte sich fort. Insbesondere Frankreich empfand die Sonderstellung des US-Dollars und damit der USA im Währungsgefüge als „exorbitantes Privileg“ und beschleunigte den Tausch seiner Dollarreserven in Gold (Eichengreen 1996: 116, 119). Der Systemkollaps in den frühen 1970erJahren war die logische Konsequenz.

\subsection{Zusammenbruch des Systems fester Wech- selkurse}

Ab April 1968 war die US-Zentralbank nicht mehr in der Lage, den Goldpreis von 35 \$ je Unze zu verteidigen, die Nachfrage nach US-Goldreserven beschleunigte sich daraufhin erheblich. Einseitige Kapitalverkehrskontrollen in Europa stellten sich als ineffektiv heraus. Auf beiderseitige Kontrollen, wie in der Bretton-Woods-Vereinbarung vorgesehen, wurde nicht zurückgegriffen; multinationalen Unternehmen fiel es zudem zunehmend leichter, Kontrollen durch konzerninterne Transaktionen zu umgehen (Helleiner 1994: 102ff). Auch die zwischenzeitliche Einführung der IWF-Sonderziehungsrechte als Anlehnung an die von Keynes vorgeschlagene globale Reservewährung 
konnte die Dollarflucht nicht mehr stoppen. Im August 1971 wurde die Goldkonvertibilität des Dollars von der amerikanischen Regierung unilateral ausgesetzt („Nixon-Schock“). Im August 1972 betrug der Goldpreis vorübergehend 70 \$; der Dollar hatte also die Hälfte seines Goldwerts im Vergleich zum fixierten Tauschverhältnis verloren.

Im März 1973 gaben Japan und die EWR-Länder die Dollarbindung ihrer Währungen auf, der Goldpreis erhöhte sich auf 90 \$. Vordergründig begünstigte dies wiederum die USA, denn durch die starke Dollarabwertung verbesserte sich ihre Leistungsbilanz (Helleiner 1994: 112-113). Mit der Entkoppelung des US-Dollar vom Goldpreis löste sich jedoch das System fester Wechselkurse, ein Grundpfeiler der BrettonWoods-Ordnung, auf. Es war ein Bruch mit jener gesamtwirtschaftlichen Ordnung, die durch Finanzmarktregulierung (seit 1933) sowie feste Wechselkurse und Kapitalkontrollen (seit 1944) geprägt war. Erhalten blieb die wirtschaftliche und politische Hegemonie der USA: War sie vor 1973 durch die Sonderstellung des Dollar als Reservewährung gegeben, so sicherten nun die zunehmende Prominenz der Finanzmärkte und deren starke Konzentration in den USA ihre globale ökonomische Vormachtstellung. Der US-Dollar behielt seinen Status als Weltwährung, in der die meisten internationalen Transaktionen und der Großteil des Rohstoff- und Warenhandels stattfanden.

\subsection{Erster Ölpreisschock und Diskreditierung des Keynesianismus}

Nachdem der Goldpreis im Sommer 1973 vorübergehend auf über 120 \$ gesprungen war, stieg der Ölpreis im September 1973 erstmals deutlich an. Ein veritabler Preisschock folgte Anfang 1974; innerhalb eines halben Jahres verdreifachte sich der Erdölpreis dadurch nahezu. Die Dollarabwertung hatte zuvor einen starken (Gold-)Preisverfall von Rohöl bewirkt, da Rohöl in USDollar gehandelt wird; dieser Preisverfall wurde durch den Rohölschock in etwa ausgeglichen. Der „Erste Ölpreisschock“ kann somit als Folge der starken Dollarabwertung verstanden werden (Schulmeister 2000: 369). Nachdem die Dollarspekulation das System fester Wechselkurse zum Einsturz gebracht hatte, stieß der Ölpreisschock die Industrieländer in die Stagflation. ${ }^{5}$

5 In Teilen der Literatur, die die Stagflation nicht als Folge des Ölpreisschocks betrachtet, erfolgt - über eine verfehlte Geldpolitik in den USA - ein direkter Bezug zwischen
Dies beschränkte die Effektivität antizyklischer Nachfragepolitik zunehmend, da sie den hohen Preisauftrieb weiter beschleunigte (Enkyo 1986: 76). Der Ölpreisschock traf die fortgeschrittenen Volkswirtschaften an einer äußerst verwundbaren Stelle, denn die Industrie war die Triebfeder der Wirtschaftsentwicklung, Dienstleistungen hatten noch keinen so hohen Stellenwert wie heute. Es war daher ein Schock für das als „Fordismus" bezeichnete Wachstumsmodell und stellte dieses gleichsam in Frage (Stockhammer 2008: 15).

\section{5. Übergang vom keynesianischen zum neolibera- len Paradigma}

Die Ölkrise schwächte die keynesianisch inspirierte Wirtschaftspolitik. Der aufkeimende Neoliberalismus unterschied sich vom Liberalismus keynesianischer Prägung dadurch, dass die Freizügigkeit der Finanzmärkte und die Freizügigkeit der Gütermärkte als komplementär betrachtet wurden (Ghosh/Qureshi 2016: 5-6; Helleiner 1994: 115-116). Die Vorstellung eines gutmeinenden Wohlfahrtsstaates wurde verworfen und durch das Bild eines Leviathanstaates, in dem die Politik durch den Druck freier Finanzmärkte diszipliniert werden müsse, ersetzt. Expansive Fiskalpolitik wurde aufgrund rationaler Erwartungen als ineffektiv oder über steigende Inflation und Zinsen als wachstumsschädlich betrachtet. Geldpolitik wurde auf die monetaristische Rolle beschränkt, das Geldmengenwachstum zu stabilisieren. Unternehmer und Industrielle, die innerhalb des Bretton-Woods-Systems von steigender Kaufkraft und wachsenden Absatzmärkten profitiert hatten, wandten sich den neoliberalen Ideen zu, da der Abbau nationalstaatlicher Kapitalbeschränkungen ihre zunehmend globalisierten Geschäftstätigkeiten begünstigte (Helleiner 1994: 119).

Mit der praktischen und theoretischen Diskreditierung des Keynesianismus kippte ein weiterer Grundpfeiler des bestehenden makroökonomischen Ordnungsrahmens. Die ideologische Zeitenwende ist an der Pfundkrise 1976 ablesbar: Sie wurde nicht durch fundamentale makroökonomische Probleme ausgelöst - das britische Leistungsbilanzdefizit nahm ab -, sondern durch die Unzufriedenheit der Investoren mit der keynesianisch agierenden Labour-Regierung (Helleiner 1994: 125). Die Unterstützung durch den IWF wurde auf Druck der USA und Westdeutschlands an

dem Fall des Festkurssystems und der gestiegenen Inflation (vgl. Barsky/Kilian 200o). 
straffe monetaristisch geprägte Sparvorgaben geknüpft. Eine Zurückweisung dieser Bedingungen hätte eine starke Beeinträchtigung des Außenhandels, den Reputationsverlust an den internationalen Finanzmärkten und unabsehbare Folgen für die Konjunktur zur Folge gehabt. Es war dem darauffolgenden "Thatcherismus“, also der neoliberalen Politik britischer Prägung, vorbehalten, dieses systematische Dilemma nationalstaatlicher Wirtschaftspolitik innerhalb einer zunehmend auf Konkurrenz abzielenden internationalen Wirtschaftsordnung aufzulösen.

\subsection{Zweiter Ölpreisschock und Hochzinspolitik}

Die US-Zentralbank verfolgte als Reaktion auf den ersten Ölpreisschock eine expansive Geldpolitik, die den Dollarkurs wieder stark sinken ließ. Durch die Dollarabwertung sollten auch andere Länder zu einer expansiven Politik angehalten werden (Eichengreen 1996: 144; vgl. auch Mihm 2010). Nachdem der Goldpreis im August 1976 auf knapp über 100 \$ zurückgegangen war, stieg er kontinuierlich an und kletterte Anfang 1979 auf über 240 \$. Im März 1979 setzte der zweite Ölpreisschock ein; der Preisanstieg dauerte diesmal länger und erfolgte parallel zur anhaltenden Dollarentwertung. Zwischen März 1979 und April 1980 stieg der Rohölpreis um das Anderthalbfache und trieb die Inflation immer weiter an. Die amerikanische Zentralbank unter ihrem neuen Gouverneur Paul Volcker reagierte mit einem Zinsschock, durch den der Dollarabfluss gestoppt wurde (vgl. The Economist 2010): Von Juli bis Dezember 1980 wurde der Leitzinssatz von $9 \%$ auf knapp 19 \% mehr als verdoppelt. Erst ab August 1981 wurde er wieder schrittweise verringert, und erst im Oktober 1982 sank er wieder unter $10 \%$.

\subsection{Geldmarktfonds}

Mit der Hochzinspolitik wurde die Finanzmarktregulierung als konstitutives Element jenes makroökonomischen Ordnungsrahmens preisgegeben, denn sie konterkarierte die Zinssatzdeckelung und provozierte Ausweichverhalten. Der zwei Jahrzehnte zuvor etablierte Eurodollarmarkt bot dafür die besten Bedingungen (s. u.). Aber auch am heimischen Markt gelang es Finanzmarktakteuren, die Zinssatzdeckelung zu umgehen, nämlich über Geldmarktfonds. Begünstigt wurde dies durch die Deregulierung der New Yorker Börse 1975 („Mayday“, Enkyo 1989: 76ff.); die Abschaffung gesetzlich geregelter Handelsgebühren schmälerte die
Profite der Finanzdienstleister und drängte sie in neue Geschäftsfelder.

Geldmarktfonds veranlagten Kapital in USStaatsanleihen und gaben die Renditen an die Anleger weiter. Da es sich dabei strenggenommen nicht um Zinserträge handelte, kam Regulation Q nicht zur Anwendung. Im Gegensatz zu anderen Anlageformen, wie etwa Unternehmensanleihen oder Aktien, war die Veranlagung in heimische Staatsanleihen aber nicht mit höherem Risiko behaftet als die Veranlagung am Sparkonto, eher mit geringerem. Geldmarktfonds generierten also höhere Renditen als Bankeinlagen bei maximal gleich hohem Risiko; die Zinssatzdeckelung wurde damit effektiv umgangen. Gegründet 1977 von Merrill Lynch waren sie eine der ersten Formen von „Schattenbanken“, also von Institutionen, die die Aufgaben von Banken wahrnahmen, ohne den Regeln, die für Banken galten, zu unterliegen (Enkyo 1989: 84-87). Im Zuge der kompetitiven Finanzmarktderegulierung wurden ihre Aktivitäten später immer weitreichender und sie mutierten zu komplexen Finanzinstrumenten, die in der Krise von 2007/08 eine prominente Rolle einnahmen (Eichengreen 2015: 177, 207).

Bis in die 1970er-Jahre war der Renditevorsprung von Staatsanleihen gegenüber Spareinlagen unerheblich, denn Bankeinlagen waren zwar durch die Zinssatzdeckelung reguliert, gleichzeitig sorgte aber „Financial Repression“ dafür, dass die Renditen auf Staatsanleihen gering waren. Der Volcker-Schock (der Zinsschock 1980-1982) hob den Prozess der kompetitiven Finanzmarktderegulierung auf eine neue Ebene: Während die Zinssatzdeckelung im Rahmen des beschränkten Wettbewerbs die Profitabilität der Bankgeschäfte erhöht hatte, wirkte sie sich im neuen Umfeld konkurrierender Anbieter, die sie umgehen konnten, abträglich aus und führte zu einem starken Kapitalabfluss von Banken zu den Geldmarktfonds (Gilbert 1986: 30). Die Divergenz zwischen Bankeinlagen und Geldmarktfonds brachte aber auch eine Benachteiligung kleiner Anleger mit sich, denen der Zugang zu Geldmarktfonds oft verwehrt blieb (Enkyo 1989: 101). Der Gesetzgeber sah sich der Aufgabe gegenüber, diese Divergenz zu beseitigen, indem er entweder

1. die Rendite von Geldmarktfonds deckelt, oder

2. die Verzinsungen von Bankeinlagen dereguliert.

Variante eins hätte die Sparer, die in Geldmarktfonds investiert waren, und den öffentlichen Haushalt belastet, da Geldmarktfonds wichtige Abnehmer von Staatsanleihen waren. Zudem hätte sie das Ziel, den Dollarabfluss zu stoppen, konterkariert. Von einer 
Deregulierung der Bankeinlagen ging hingegen kein unmittelbares Konjunkturrisiko aus. Die Sorge um die Finanzmarktstabilität, die 1933 zur Zinssatzdeckelung geführt hatte, rückte angesichts des Zusammenbruchs des wirtschaftlichen Ordnungsrahmens, in dem die Finanzmarktregulierung eingebettet war, in den Hintergrund. Zwar waren die Risiken, die mit der Finanzmarktderegulierung einhergingen, durchaus bekannt; sie wurden aber von der Zuversicht, die von den boomenden Finanzmärkten ausging, überdeckt (Mayer 2015: 188). Mit dem Depository Institutions Deregulation and Monetary Control Act (DIDMCA) beschloss der amerikanische Gesetzgeber 1980, Regulation Q bis 1986 vollständig auslaufen zu lassen.

\section{Kompetitive Finanzmarktderegulierung}

Mit diesem Gesetz gewann der legislative Prozess der kompetitiven Finanzmarktderegulierung, der bis zur Finanzmarktkrise 2007 anhielt, an Schwung. Wettbewerbsvorteile, die sich für einen Teil der Finanzbranche aus der Umgehung bestehender Regulierungen ergaben, wurden durch Deregulierungen ausgeglichen (Stockhammer 2013: 514f.). Die mögliche Alternative zu dieser Vorgehensweise, nämlich eine stärkere Regulierung der im Wettbewerb bevorzugten Gruppe, setzte sich mangels Koordinationsfähigkeit bzw. Kooperationswilligkeit auf internationaler Ebene („kooperative Re-Regulierung“) nicht durch; Deregulierung erwuchs hingegen aus einzelstaatlichen Initiativen.

Dies zeigte sich am Beispiel des Eurodollarmarkts, der in der Anfangsphase der kompetitiven Deregulierung eine entscheidende Rolle spielte: Seine Freizügigkeit, die im alten Ordnungsrahmen die Dollarnachfrage stärkte ohne konjunkturell restriktiv $\mathrm{zu}$ wirken, benachteiligte nun die Finanzwirtschaft in den USA, die nach wie vor der Zinssatzdeckelung unterlag (Helleiner 1994: 135ff.). Der Versuch der USA, den Eurodollarmarkt zu re-regulieren, scheiterte am Widerstand jener Staaten, die von ihm durch die Stärkung ihrer jeweiligen Finanzzentren profitierten: Großbritannien (London) und die Schweiz (Zürich). Während also kompetitive Deregulierung durch einzelstaatliche Handlungen in Gang gehalten wurde, scheiterte die Re-Regulierung an der mangelnden Kooperationswilligkeit der Länder; zumal im neuen Ordnungsrahmen flexibler Wechselkurse und zunehmend freien Kapitalverkehrs die Notwendigkeit kooperativen Verhaltenes (d. h. kooperativ gegenüber der hegemonialen US-Politik) abnahm.

\subsection{Deregulierung entfesselt die Finanzwirt- schaft}

Da die Re-Regulierung des Eurodollarmarkts scheiterte, wurden in den USA 1981 „internationale Bankfazilitäten" (IBF) als explizite Konkurrenz zum Eurodollarmarkt zugelassen (Helleiner 1994: 138). IBF waren Zweiggesellschaften von US-Banken, deren Einlagen- und Kreditgeschäft nur US-Ausländern offenstand und die von der Einlagensicherung, den Mindestreservevorschriften und von Besteuerung befreit waren. Als direkte Konkurrenz zum Eurodollarmarkt schwächten IBF den Finanzplatz London. Dieser wurde zudem von den Wellen, die die Nachbeben des Volcker-Schocks schlugen, erfasst: Der Zinsschock in den USA löste eine Schuldenkrise in Lateinamerika aus; er verteuerte den Schuldendienst der zu einem beträchtlichen Teil in variabel verzinsten Dollaranleihen verschuldeten Länder signifikant. Die Dollaraufwertung verschlimmerte deren Liquiditätslage. Die britischen Banken waren die wichtigsten Player im internationalen Bankgeschäft und wurden von der lateinamerikanischen Schuldenkrise besonders hart getroffen (Enkyo 1989: 191).

Diese beiden Einflussfaktoren, die Einführung der IBF in den USA und die lateinamerikanische Schuldenkrise, die mit dem Zusammenbruch des Bretton-Woods-Systems und der einsetzenden Finanzmarktderegulierung ursächlich in Zusammenhang stehen, gingen der Liberalisierung der Finanzmärkte in Großbritannien ab 1983 voran. Ähnlich den MaydayReformen in den USA 1975 wurde v. a. der Wertpapierhandel durch die Abschaffung der Börsengebühren liberalisiert. Dies bewirkte eine Beschleunigung an Zusammenschlüssen, Konsolidierungen und Beteiligungen ausländischer Finanzunternehmen. Die entsprechenden Gesetzesänderungen, der „Big Bang“, traten 1986 in Kraft (Enkyo 1989: 203f., 210). Die Einführung von IBF in den USA beschleunigte nicht nur den Deregulierungsprozess im Ausland, sie öffnete auch das Tor zu weiteren Deregulierungsschritten im Inland: Mit dem Garn-St. Germain Depository Institutions Act (GGDIA) von 1982 wurden den Bausparkassen neue Betätigungsfelder eröffnet, insbesondere die Unternehmensfinanzierung (Sherman 2009: 7). Der GGDIA war eine Reaktion auf die Abschaffung der Zinssatzdeckelung durch den DIDMCA, durch die den Bausparkassen die Zinsvorteile abhandengekommen waren. Die Bevorzugung der „savings and loans associations“ in Regulation Q war ursprünglich als Förde- 
rung von Bauinvestitionen konzipiert gewesen (Gilbert 1986: 28).

Während die Einführung der IBF prototypisch für die kompetitive Deregulierung auf internationaler Ebene war, war es der GGDIA für die innerstaatliche Deregulierung: Die Bausparkassen, deren Vorteile durch Deregulierung endeten, wurden entlastet, indem ihr Betätigungsfeld erweitert und ihnen neue Geschäftsmöglichkeiten eröffnet wurden. Gleichzeitig schränkte dies die Profitabilität der in diesen Bereichen angestammten Unternehmen ein und erzeugte neue Risiken (Sherman 2009: 7). Die „Big Bang“- und „Mayday“Reformen entzogen den Investmentbanken einen gesicherten Strom an Einkünften (Beratungsgebühren) und exponierten sie der direkten Konkurrenz zu anderen Finanzdienstleistern. Die kleinteiligen Personengesellschaften waren nicht mehr wettbewerbsfähig und wurden durch kapitalgesellschaftliche Organisationsformen oder Zusammenschlüsse mit anderen Banken ersetzt (The Economist 2008: 75-76).

\subsection{Megafusionierungen: Die zunehmende Vormachtstellung des Finanzkapitals}

Die Aufhebung der Zinssatzdeckelung hatte also eine zunehmende Überlappung der Geschäftsfelder von Banken, Bausparkassen und Geldmarktfonds zur Folge. Dies stellte wiederum die strikte Spartentrennung, die neben der Zinssatzdeckelung ein weiterer Grundpfeiler des Banking Act 1933 war, in Frage. Die vagen Formulierungen des Gesetzestextes ließen der USZentralbank einen hohen Interpretationsspielraum, den sie im Lichte der neuen makroökonomischen Rahmenbedingungen für eine weitere Deregulierung nutzte. 1986 wurde der Glass-Steagall-Act neu ausgelegt und Geschäftsbanken der Einstieg in Wertpapiergeschäfte im Ausmaß von maximal fünf Prozent ihrer Gesamterträge ermöglicht (Sherman 2009: 9). Zentralbankgouverneur Volcker stand der Neuauslegung skeptisch gegenüber. Gegen seine Stimme wurde es Geschäftsbanken ermöglicht, Unternehmensanleihen, hypothekenbesicherte Schuldverschreibungen und Gemeindeschuldverschreibungen zu begeben. Mit der Wahl des prononcierten Deregulierungsbefürworters Alan Greenspan zum Zentralbankgouverneur 1987 setzte sich die Aushöhlung der Spartentrennung fort. Erst wurde die Obergrenze für Wertpapiergeschäfte auf $10 \%$ der Gesamterträge erhöht, später (1996) auf $25 \%$, was einer faktischen Abschaffung der Spartentrennung gleichkam. Der Großteil der faktischen Deregulierung
- weniger Kreditlenkung, Reduktion von Reservevorschriften, Abschaffung von Zinsobergrenzen, Verringerung von Markteintrittsbarrieren, Abschaffung von Kapitalverkehrskontrollen - war in den Industrieländern bis Mitte der 1990er-Jahre umgesetzt, wie der Finanzmarktliberalisierungsindex von Abiad/Detragiache/Tressel (2010: 291, Abb. 1) ${ }^{6}$ zeigt.

Dieses neue Umfeld weitreichend deregulierter Finanzmärkte, insbesondere die Aushöhlung der Spartentrennung zwischen 1986 und 1999, löste eine Welle an Unternehmenszusammenschlüssen in der Finanzwirtschaft aus; zwischen 1990 und 1998 reduzierte sich die Anzahl an Banken in den USA um fast ein Drittel. 1998 erfolgte die bis dahin größte Fusion zweier Unternehmen, jene zwischen „Travelers Insurance Group“, einem Versicherungsunternehmen, und „Citicorp“, dem Mutterkonzern von „Citibank“, einer Bank. Gemäß den Vorschriften des Glass-Steagall-Act hätte das neu entstandene Finanzkonglomerat, "Citigroup“, die Versicherungssparte innerhalb von zwei Jahren abtreten müssen (Sherman 2009: 9-10). Dies geschah jedoch nicht; stattdessen wurden gemäß der Deregulierungslogik die Gesetze geändert: Der Glass-SteagallAct wurde 1999 durch den Financial Modernization Act (auch: „Gramm-Leach-Bliley-Act") ersetzt und das Verschmelzen unterschiedlicher Sparten (Investment-, Makler- und Versicherungsaktivitäten) mit dem normalen Einlagengeschäft, das Geschäftsbanken vorbehalten war, legitimiert. Die gesetzliche Abschaffung der Spartentrennung war ein weiterer Meilenstein im Prozess der Finanzmarktderegulierung und ein Wegbereiter der Finanzmarktkrise 2007/08. Caprio/ Honohan/Stiglitz (1999) fassten die bis dahin erfolgte Liberalisierung der Finanzmärkte in drei Kategorien zusammen:

- Abschaffung von Zinssatz- und anderer Preiskontrollen,

- Privatisierung von Finanzdienstleistungen und Reduktion der Kreditlenkung durch den Staat,

- Zulassung neuer Marktteilnehmer, Abbau von Branchenbarrieren und Beseitigung gesetzlicher Bestimmung zur Verhinderung von Wettbewerb.

6 Der Index misst den Grad der Finanzmarktliberalisierung anhand von sieben Dimensionen - Kreditkontrollen, Zinssatzkontrollen, Marktzugangsbeschränkungen, Staatseigentum, Kapitalverkehrskontrollen, Finanzmarktaufsicht, Wertpapiermärkte - in 91 Ländern im Zeitraum von 1975 bis 2005. 
6.3 Financial Engineering am Höhepunkt der Deregulierung

In den 2oooer-Jahren verfestigte sich die Vormachtstellung der Finanzwirtschaft innerhalb des zunehmend nach neoliberalen Gesichtspunkten umgestalteten marktwirtschaftlichen Ordnungsrahmens weiter: Nachdem die Deregulierung in den 1980erJahren mit dem Auslaufen der Zinssatzdeckelung in Schwung gekommen war und sich in den 1990er-Jahren mit der Aushöhlung der Spartentrennung beschleunigt hatte, brachten die 2oooer-Jahre den Aufstieg der (unregulierten) Derivate. Wie die Genese der Deregulierungsmaßnahmen wurzelt auch die der Derivate in den Trümmern des Bretton-Woods-Systems: Die Wechselkursturbulenzen, die dessen Zusammenbruch erzeugte, verliehen den als Absicherung konzipierten Zins- und Währungsswaps einen wichtigen Auftrieb (The Economist 2008: 75). So komplex und intransparent Derivate in den 2oooer-Jahren wurden, so schlicht und einfach ist ihr Grundprinzip: nämlich den Wert aus der Wertentwicklung eines anderen Produktes oder aus anderen Vermögenspreisen in einer systematischen Weise abzuleiten bzw. zu „derivieren“. Ein Swap ist ein Tauschgeschäft $\mathrm{zu}$ einem vereinbarten zukünftigen Zeitpunkt, bei dem der Zinssatz oder der Wechselkurs für den Käufer fixiert wird; d. h. sein Wert ist abhängig von der künftigen Zinssatz- bzw. Wechselkursentwicklung.

Entworfen als Versicherung kann ein Derivat ebenso gegenteilig eingesetzt werden, also spekulativ. Durch den im Jahr 2000 beschlossenen Commodity Futures Modernization Act wurden Derivate explizit von der Regulierung ausgenommen (Sherman 2009: 11). Ihr Handel blieb „over the counter“, also bilateralen Vereinbarungen ohne Kontroll- oder Clearingins$\tan z$ überlassen. Dies entsprach der neoliberalen Idee größtmöglicher Effizienz, da unterstellt wurde, dass die Händler über umfassende Informationen verfügten und durch Risikoabwägung stabilitätswahrend handelten (Mayer 2015: 190). Der bilaterale, unkontrollierte Handel nährte jedoch Wildwuchs und erzeugte Intransparenz; stabilisierende Transaktionen sind gerade bei einer durch Herdenverhalten getriebenen Marktentwicklung oft nicht profitabel (Shiller 2000: 151, 172). Als Zins- und Wechselkursinstrumente legten Swaps in den 1970er-Jahren den Grundstein für den späteren Höhenflug von Derivatprodukten im Zuge der voranschreitenden Finanzmarktderegulierung; als Kreditausfallsversicherungen („credit default swaps“) spielten sie eine Schlüsselrolle in der Finanzmarktkrise 2007/o8 (The Economist 2008: 76-77).

\subsection{Verquickung finanzwirtschaftlicher Instru- mente}

Ohne die Entwicklungen im Rahmen der kompetitiven Deregulierung seit den 1980er-Jahren wäre die Finanzmarktkrise in ihrer Ausprägung von 2007/08 nicht möglich gewesen. Dies zeigt sich am besten am Geschäftsmodell jener Finanzkonglomerate, die im Zuge der Finanzmarktderegulierung entstanden waren und sich ab 2007 im Zentrum der Krise befanden. Wilmarth (2009: 994) benennt 17 Finanzkonglomerate („large complex financial institutions"); darunter 11 „Universalbanken“, also Unternehmen, die als ursprüngliche Geschäftsbanken gleichzeitig Wertpapiergeschäfte tätigten. Dazu zählten in den USA die Bank of America, JP Morgan Chase, Citigroup und Wachovia, in Großbritannien Barclays, Royal Bank of Scotland und HSBC, in der Schweiz Credit Suisse, in Deutschland die Deutsche Bank und in Frankreich BNP Baripas und Société Générale. Darüber hinaus zählten zu den großen Finanzkonglomeraten von 2007 die fünf US-Investmentbanken Bear Stearns, Goldman Sachs, Lehman Brothers, Merrill Lynch und Morgan Stanley; durch ihr Einlagengeschäft waren sie auch als Geschäftsbanken tätig. Der Versicherer American International Group (AIG) war schließlich intensiv im Derivatgeschäft involviert. Das Geschäftsmodell dieser Finanzkonglomerate wird als „originate to distribute" bezeichnet (Wilmarth 2009: 969, 981). Finanzdienstleistungen, die im Rahmen der Finanzmarktregulierung strikt voneinander getrennt waren, werden zusammengefasst und so in einen zusammenhängenden Kreislauf gebunden:

1. Kreditvergabe,

2. Kreditverbriefung,

3. Kreditverbriefungsderivate,

4. Vertrieb der Wertpapiere.

In dieser Sequenz von Finanzdienstleistungen ist die Vermischung der vormals getrennten Sparten evident: Die Kreditvergabe (Punkt 1) war im Rahmen regulierter Finanzmärkte die ausschließliche Aufgabe von Geschäftsbanken; der Wertpapierhandel (Punkt 4) ausschließliches Betätigungsfeld der Investmentbanken. Am Anfang und am Ende des für die Finanzkrise maßgeblichen „originate to distribute“-Modells standen also Finanzdienstleistungen, die ursprünglich durch die Spartentrennung explizit voneinander getrennt waren. 
Durch die Kombination dieser beiden Dienstleistungen wurde Punkt 2, die Kreditverbriefung, also die Erzeugung von Wertpapieren auf Basis von Kreditgeschäften, ermöglicht. Kreditverbriefungen wie „Asset Backed Securities“ (ABS) und „Collateralized Debt Obligations“ (CDOs) standen im Zentrum der Finanzmarktkrise 2007/08 (Eichengreen 2015: 75-77, 100). Potenziert wurde ihre Wirkung durch den spekulativen Einsatz von Kreditverbriefungsderivaten (v. a. „Credit Default Swaps“, CDS), die die Verschränkung des Kredit- und des Wertpapiergeschäfts über die Kreditverbriefung hinaus erweiterten.

Bankeinlagen sind die günstigste Form der Finanzierung, nicht zuletzt da sie wegen der Einlagensicherung vergleichsweise geringe Zinskosten verursachen (Wilmarth 2009: 978). Gleichzeitig bilden die Kredite, die auf Basis der Einlagen vergeben werden, die Geschäftsgrundlage für die äußerst rentablen Verbriefungen. So können die Erträge von Investmentbanken erwirtschaftet werden, ohne auf Kapitalmarktfinanzierung beschränkt zu sein. Durch die Erstellung von Derivaten werden darüber hinaus die Erträge von Versicherungen abgeschöpft. Indem sie sich der Finanzierungsstruktur von Geschäftsbanken und der Vermögensstrukturen von Investmentbanken und Versicherungen bedienten, kombinierten die Finanzkonglomerate diese drei Formen von Finanzdienstleistungen durch „originate to distribute“ also in optimaler Weise.

Aus gesamtwirtschaftlicher Sicht ergibt sich durch die Verquickung von Wertpapier- und Einlagengeschäft hingegen genau jene verheerende Wirkung, die die Krisen 1929-1933 und 2007/o8 zu Weltwirtschaftskrisen mit sehr hohen öffentlichen und sozialen Kosten machte. Die Verzahnung unterschiedlicher Formen von Finanzdienstleistungen durch dominante Finanzkonglomerate, welche aufgrund der Deregulierung der Finanzmärkte entstanden waren, ermöglichte erst die Finanzmarktkrise von 2007/08.

\section{Nachtrag: Die Rolle der Geldpolitik}

Als Ursache der Finanzmarktkrise 2007/o8 wird oft die expansive Geldpolitik in den USA ab 2001 genannt, mit der die Zentralbank auf einen Aktienkursrutsch und einen Konjunkturabschwung reagierte (vgl. Taylor 2012; Breuss 2009). Sie stand auch im Zeichen der Entwicklungen in Japan, das sich seit Kurzem in der Deflation befand, was die amerikanische Zentralbank unbedingte vermeiden wollte (Eichengreen 2015: 84).
Darüber hinaus zielte die Geldpolitik genau darauf ab, was ihr später zum Vorwurf gemacht wurde, nämlich ihre expansive Wirkung über den Vermögenskanal, also durch Ankurbelung der Vermögenspreise, zu entfalten (Wilmarth 2009: 1005). Die in diesem Beitrag skizzierte Geschichte der Finanzmarktderegulierung in den USA zeigt, dass sich das makroökonomische und regulatorische Umfeld in den frühen 20ooer-Jahren ganz erheblich von früheren Phasen der konjunkturellen Unterauslastung unterschied. Das enorme Gewicht, das die Finanzmärkte im gesamtwirtschaftlichen Kreislauf nunmehr einnahmen, wurde zu einem Hindernis für eine Geldpolitik, die auf eine Glättung des realwirtschaftlichen Konjunkturzyklus abzielte. Die Finanzmarktkrise wurde erst durch die Präsenz großer Finanzkonglomerate, durch die massive Spekulation mit Finanzderivaten sowie die Verzahnung von Einlagen-, Wertpapier- und Derivatgeschäft ermöglicht. Die US-Zentralbank ist daher weniger für die lockere Geldpolitik zu kritisieren, die im Rahmen der heimischen und weltwirtschaftlichen Umstände am Beginn der 20ooer-Jahre durchaus nachvollziehbar war, sondern vielmehr für die anhaltende Deregulierung der Finanzmärkte, die sie über Jahrzehnte zugelassen und aktiv betrieben hatte.

7 Eine frühere oder stärkere Straffung der Geldpolitik hätte zudem dazu führen können, die Kapitalzuflüsse zu beschleunigen und dadurch die spekulative Blase weiter $\mathrm{zu}$ befeuern. 


\section{Literatur}

Abiad, A./Detragiache, E./Tressel, T. (2010): A New Database of Financial Reforms. IMF Staff Papers. International Monetary Fund (IMF), 57 (2).

Barsky, R./Kilian, L. (2000): A Monetary Explanation of the Great Stagflation of the 1970s. NBER Working Paper, (7547). Online: http://www.nber.org/papers/w7547.pdf [Mai 2016].

Battilossi, St. (2005): The Second Reversal: The Ebb and Flow of Financial Development in Western Europe, 1950-1991. Madrid: Department of Economic History and Institutions, Universidad Carlos III.

Beirat für Wirtschafts- und Sozialfragen (2009): Österreich und die internationale Finanzkrise. Wien: Ueberreuter, (83).

Bentley, K. (2015): The 2008 Financial Crisis: How Deregulation Led to the Crisis. Senior Theses. Online: http://publications.lakeforest.edu/seniortheses/55 [Mai 2016].

Bhidé, A. (2009): An Accident Waiting to Happen. Critical Review: A Journal of Politics and Society, 21 (2-3), 211-247.

Bordo, M.D. (2017): The Operation and Demise of the Bretton Woods System; 1958 to 1971. NBER Working Paper, (23189). Online: http://www.nber.org/papers/w23189 [Februar 2017].

Breuss, F. (2009): The Financial Crisis as an Overshooting Phenomenon. WIFO Working Papers, (336).

Caprio, G./Honohan, P./Stiglitz, J.E. (1999): Financial Liberalization: How Far? How Fast? Introduction and Overview. Online: http://www.econ.chula.ac.th/public/members/ sothitorn/liberalization_2.pdf [Mai 2016].

Der Spiegel (1961): Angst vor dem Run. Der Spiegel, (10), 18-25.

Eichengreen, B. (1996): Globalizing Capital - A History of the International Monetary System. Princeton: Princeton University Press.

Eichengreen, B. (2007): The European Economy since 1945 Coordinated Capitalism and Beyond. Princeton: Princeton University Press.

Eichengreen, B. (2015): Hall of Mirrors - The Great Depression, the Great Recession, and the Uses - and Misuses - of History. New York: Oxford University Press.

Enkyo, S. (1989): Financial Regulation and International Safeguards. PhD Thesis. London: London School of Economics.

Friedman, M. (1953): The Case for Flexible Exchange Rates, In: Essays in Positive Economics, University of Chicago Press, 157-203.

Ghosh, A. R./Qureshi, M. S. (2016): What's In a Name? That Which We Call Capital Controls. IMF Working Paper, WP/16/25. Online: https://www.imf.org/external/pubs/ $\mathrm{ft} / \mathrm{wp} / 2016 / \mathrm{wp1625}$.pdf [Mai 2016]

Gilbert, R. A. (1986): Requiem for Regulation Q: What It Did and Why It Passed Away. Federal Reserve Bank of St. Louis Review, February, 22-37.
Handler, H. (2008): Vom Bancor zum Euro. Und weiter zum Intor? WIFO Working Papers, (317). Online: http:// www.wifo.ac.at/publikationen/working_papers?detailview=yes\&publikation_id=31986 [Mai 2016].

Helleiner, E. (1994): States and the Reemergence of Global Finance - From Bretton Woods to the 1990s. Ithaca: Cornell University Press.

Helleiner, E. (2010): A Bretton Woods moment? The 20072008 crisis and the future of global finance. International Affairs, 86 (3), 619-636.

Kotz, D. M. (2013): Changes in the Postwar Global Economy and the Roots of the Financial Crisis. In: Wolfson, M. H./ Epstein, G. A. (Hg.): The Handbook of the Political Economy of Financial Crises. New York: Oxford University Press, 395-410.

Levine, R. (2012): The Governance of Financial Regulation: Reform Lessons from the Recent Crisis. International Review of Finance, 12 (1), 39-56.

Mayer, C. (2015): Big Bang: new beginning or beginning of the end? Oxford Review of Economic Policy, 31 (2), 186-198.

Mihm, A. (2010): Obama, Carter und die Konjunkturlokomotive. Frankfurter Allgemeine Zeitung, 26.6.2010, Online: http://www.faz.net/aktuell/wirtschaft/konjunktur/ sparpolitik-obama-carter-und-die-konjunkturlokomotive-1997011.html [Mai 2016].

Ramskogler, P. (2015): Tracing the origins of the financial crisis. OECD Journal: Financial Market Trends, 2014 (2), 47-61.

Reinhart, C. M./Sbrancia, M. B. (2015): The liquidation of government debt. Economic Policy, 30 (82), 291-333.

Ruggie, J. G. (1982): International regimes, transactions, and change: embedded liberalism in the postwar economic order. International Organization, 36 (2), 379-415.

Schuberth, H. (2013): Finanzmarktregulierung in der Europäischen Union - Fünf Jahre nach Lehman. Wirtschaft und Gesellschaft, 39 (4), 509-543.

Schulmeister, St. (2000): Globalization without global money: the double role of the dollar as national currency and world currency. Journal of Post Keynesian Economics, 22 (3), 365-395.

Sherman, M. (2009): A Short History of Financial Deregulation in the United States. Washington: Center for Economic and Policy Research (CEPR).

Shiller, R. J. (2000): Irrational Exuberance. Princeton: Princeton University Press.

Silvers, D. (2013): Deregulation and the New Financial Architecture. In: Wolfson, M. H./Epstein, G. A. (Hg.): The Handbook of the Political Economy of Financial Crises. New York: Oxford University Press, 430-446.

Stiglitz, J.E. (2009): The Anatomy of a Murder: Who Killed America's Economy? Critical Review: A Journal of Politics and Society, 21 (2-3), 329-339

Stockhammer, E. (2008): Finanzkrise: Chronologie, Ursachen und wirtschaftspolitische Reaktionen - Kasinokapitalismus mit staatlichen Fremdheilungskräften. grundrisse-zeitschrift für linke theorie \& debatte, 28, 13-22. 
Stockhammer, E. (2013): Financialization and the Global Economy. In: Wolfson, M. H./Epstein, G. A. (Hg.): The Handbook of the Political Economy of Financial Crises. New York: Oxford University Press, 512-525.

Taylor, J. B. (2012): The Great Deviation, In: Koenig, E./Leeson, R./Kahn G. (Hg.): The Taylor Rule and the Transformation of Monetary Policy. Stanford, CA: Hoover Institution Press, 163-172.

The Economist (2008): Link by link - A short history of modern finance. The Economist, 389 (8602), 75-77.

The Economist (2010): Who beat inflation? The Volcker recession. The Economist, Free Exchange Economics, Online: http://www.economist.com/blogs/freeexchange/2010/03/volcker_recession [Mai 2016].

Tichy, G. (2014): Banken- und Staatsschuldenkrise: Ursachen, Folgen, Lösungsansätze, In: Hilpold, P./Steinmair, W. (Hg.): Neue europäische Finanzarchitektur - Die Reform der WWU. Berlin/Heidelberg: Springer-Verlag, 223-247.

Url, Th. (2009): Finanzmarktkrise: Entstehung, kurzfristige Reaktion und langfristiger Anpassungsbedarf. WIFOMonatsberichte, 82 (12), 909-931.

Wilmarth, A. E. jr. (2009): The Dark Side of Universal Banking: Financial Conglomerates and the Origins of the Subprime Financial Crisis. Connecticut Law Review, 41 (4), 963-1050.

Wolfson, M. H. (2013): An Institutional Theory of Financial Crises. In: Wolfson, M. H./Epstein, G. A. (Hg.): The Handbook of the Political Economy of Financial Crises. New York: Oxford University Press, 172-190. 